must be made on the basis of inadequate data, seems to have been overlooked in many contributions. And several authors refer to alternatives to PVA but fail to point out that the only realistic alternative, human judgement and intuition, is inaccurate.

Deficiencies in data are a serious problem for PVAs of endangered species, as several authors point out. They discuss various remedies, including eliminating problems in data collection, separating the effects of sampling variation and intrinsic variation, and the use of bayesian methods to incorporate uncertainties about parameter values into predictions. However, the use of data from related species receives little attention. Such data may be particularly valuable for variances of input parameters. For example, environmental variation is similar across a range of herbivore taxa (J.-M. Gaillard et al. Annu. Rev. Ecol. Syst. 31, 367-393; 2000). Furthermore, analyses of long-term data sets to estimate the frequency and severity of catastrophes and to ask whether they differ widely across taxa would be highly desirable.

The way to improve PVA is clear, but receives little attention here. It involves cycles of building models, making predictions, testing them, improving the models, and so on, as for all fields involving complex systems. The emphasis in PVA has been on building models, but the testing phase has only just begun. Much more testing is required if the field is to advance swiftly and rationally, as is happening with climate modelling.

Despite some limitations, this volume should serve as a major reference book on PVA for professional scientists, advanced undergraduates and graduate students in conservation biology.

Richard Frankham is in the Department of Biological Sciences, Macquarie University, Sydney, New South Wales 2109, Australia.

\section{Shock results}

\section{Surprise, Uncertainty and Mental Structures \\ by Jerome Kagan \\ Harvard University Press: 2002. 259 pp. $\$ 29.95, £ 20.50$}

\section{Peter Bryant}

Developmental psychology — or at any rate the part that deals with children's intellectual abilities - is in an awkward position at the moment because it keeps on producing paradoxical and contradictory results. On one hand, a series of ingenious experiments has apparently shown that babies less than one year old possess some remarkable abilities. According to these experiments, infants can discriminate numbers, can add and subtract, and can distinguish cause and effect in both the physical and the social worlds. On the other hand, a great deal of research on much older children, between the ages of three and about eight years, has apparently demonstrated that children in this age band often have serious difficulties with these same concepts. Their understanding of number appears fragile in such studies, their reasoning about arithmetical operations such as addition and subtraction is often erroneous, there are serious limitations to their grasp of cause and effect, and so on.

The contrast and apparent conflict between these two lines of research are stark and clear. Any suggestion that 6-month-old babies are much cleverer than children four and five years their senior would be highly implausible, and would be anathema to most developmental psychologists - their subject is dedicated to the proposition that intellectual skills improve throughout childhood. Another possible solution to this apparent impasse is to reappraise the work on infants. Are they as smart as this research suggests?

Jerome Kagan, who is a distinguished US developmental psychologist with an interest in children's emotional and intellectual development, has now provided such a reappraisal in his new book. He is well placed to do so because much of his research has been on children's expectations and their reactions to congruous and incongruous events. Nearly all of the work that apparently demonstrated various remarkable abilities in infants used their reaction to novelty and their surprise at unexpected events as a way of measuring such abilities. If, for example, you add one object to another and then show the baby that the product of your actions is three objects, and if the baby is more surprised to see this as the result than she is to see two objects there, you can argue that the baby knows something about addition.

Kagan's discussion of this pervasive use of babies' reactions to novel and unexpected events comes in the second half of the book. It is based on ideas, which he expounds in the first part, about the ways in which children and adults form expectations and are surprised when these are not fulfilled. His reasoning is sophisticated and interesting, but his conclusions are easy to summarize. $\mathrm{He}$ is highly sceptical of the claims made about the presence of surprising abilities in infants, and argues that their achievements in these studies can be explained without ascribing huge intellectual acumen to them.

Kagan's reassessment of research on infants is the most important part of his new book. His arguments on the subject are controversial, of course, and should provoke a much-needed discussion. So the book will make a real contribution to the debate, and is definitely worth reading.

Peter Bryant is in the Department of Experimental Psychology, University of Oxford,

Oxford OX1 3UD, UK.

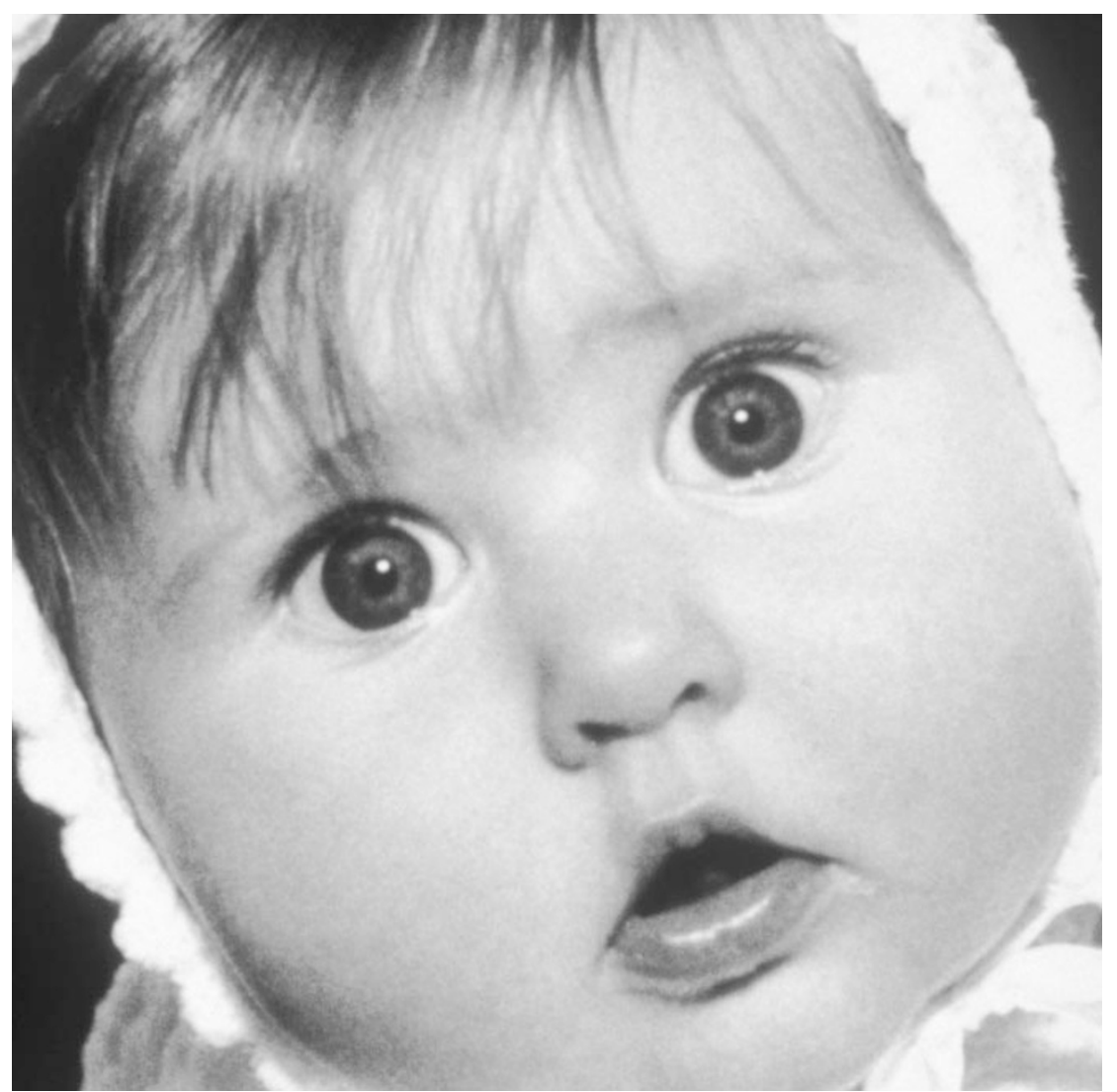

Bright eyes? Babies' responses to surprising results may not be a good guide to their intelligence. 